\title{
Risk Factors for Acquisition of Multiply Drug-Resistant Gram-Negative Bacteria
}

\author{
I.M. Gould
}

\begin{abstract}
Some bacteria are naturally resistant to many antibiotics and most can become multiply resistant. Multiply resistant gram-negative bacteria have proved a particular problem over the last 30 years, but the development of new agents has lessened their significance for most clinicians. Now, however, clinical practice is threatened by the lack of new classes of antibiotics, the widespread emergence of resistance and the advent of plasmid-mediated cephalosporinases by which the spread of resistance is likely to be rapid. Increased use of prophylaxis in immunosuppressed and intensive care patients is likely to aggravate the problem, as is the use of new broad-spectrum agents in the community. More directed and restricted antibiotic use and better education of patients and prescriber are necessary to contain the problem of antibiotic resistance. Improved surveillance of sensitivity trends is essential. Many outbreaks also are associated with poor infection control techniques. The cost of outbreaks due to multiply resistant organisms and lack of compliance with infection control procedures needs to be properly studied. While many predisposing factors for the acquisition of these organisms and the development of infection are understood, the multifactorial nature of illness in many patients complicates the issue, necessitating further study of risk factors and preventative and therapeutic measures.
\end{abstract}

With the advent of penicillin, erythromycin and then the semisynthetic penicillins, the problems of staphylococcal hospital infection largely receded to be replaced in the 1970 s by infection due to resistant gram-negative bacilli. Many of these organisms, such as Pseudomonas aeruginosa, were innately resistant to all but one or two of the then available antibiotics. With the development of aminoglycosides, acylureido-penicillins, expanded-spectrum cephalosporins, and then the monobactams, 4-fluoroquinolones and carbapenems, the perception was that the major resistance problems had been overcome. Kresken and Wiedemann (1) wrote in 1986 that there were few bacteria with resistance to nearly all the drugs that were then available. They thought that the reasons for this were probably infection control measures, rational use of antibiotics in hospitals and the introduction of new drugs with broad-spectrum activity. Gram-positive infections such as those due to methicillin-resistant staphylococci, penicillinand glycopeptide-resistant enterococci and $\mathrm{J} \mathrm{K}$

Department of Clinical Microbiology, Aberdeen Royal Infirmary, Aberdeen AB9 2ZD, UK. coryneform bacilli were thought to be the potential problem organisms of the 1990s.

While these problems of resistant gram-positive organisms still remain with few potential solutions in sight, the realisation is that multiply resistant gram-negative bacilli (MRGNB) are a recurrent problem. Aminoglycoside resistance is widespread, with little development of new agents in this class, 4-fluoroquinolone resistance is already common, particularly in Pseudomonas spp., and resistance develops readily despite the lack of demonstrated plasmid-mediated mechanisms. Inducible chromosomal cephalosporinases are now commoniy manifest in inducible Enterobacteriaceae (Citrobacter, Enterobacter, Serratia and Morganella spp.) even in the absence of cephalosporin treatment (2), and, most worryingly, modification of the ubiquitous SHV-1 and TEM-1 and TEM-2 enzymes of Enterobacteriaceae now determine monobactam and third-generation cephalosporin resistance (3). Moreover, although carbapenemases are still rare, 4-fluoroquinolone use can determine broad-spectrum quinolone and carbapenem resistance through permeability changes (4), and enzyme-mediated resistance to aminoglycosides and third-genera- 
tion cephalosporins is described increasingly on single plasmids (5).

With this worrying situation, which shows no sign of being solved by the development of new agents, it is important that we understand fully the epidemiology of MRGNB infection in order to prevent such infections. This article summarises current knowledge on this issue and offers indications for further research.

\section{Epidemiology of Multiply Drug-Resistant Bacteria}

Cohen (6) has summarised the main risk factors for acquisition of resistant organisms. Clearly, the organism itself is important, and gram-negative bacteria, with their greater barrier to permeability than gram-positive bacteria, in addition to a greater diversity of plasmid and chromosomal enzymes, probably have greater potential to become multiply resistant. Hospitals, and particularly intensive care units (ICU), are important environmental factors, encouraging not only emergence and persistence of resistance but also providing frequent opportunities for transmission of resistant organisms from patient to patient. Reservoirs of both pathogenic organisms and genetic material are clearly important. The human gut and skin are large reservoirs, and hospitals also provide many inanimate reservoirs such as water and food supplies and re-usable liquids. It is not within the scope of this article to discuss animal reservoirs and their relevance to human antibiotic resistance. Suffice to say that the contribution of animals (and antibiotic use in them) is probably minor in overall terms compared with the use of antibiotics in humans (7).

Societal behaviour, particularly increased sexual promiscuity, clearly has allowed increased dissemination of resistant venereal diseases, as have technological changes by allowing increased travel and successful treatment of heavily immunosuppressed patients. There have also been major demographic changes in the population. The elderly are more susceptible to infection, and increased use of elderly and day care facilities encourages emergence and or transmission of resistant organisms. However, the lack of technology and economic wealth in urban areas of developing countries leads to transmission of many infectious diseases due to poverty and poor sanitation. As in the developed world, many of these infections are caused by multiply resistant organisms following widespread antibiotic use and abuse, e.g. the newly described 0139 vibrio causing the latest cholera epidemic (8). Over-the-counter or black market availability of many of these agents undoubtedly aggravates resistance problems (9, 10). Although there are many resistance problems associated with community-acquired organisms, the problem with MRGNB is much more associated with hospitals and nursing homes. McGowan et al. (11) addressed this issue by comparing the sensitivity of various isolates from hospitals and the community. In general, comparing hospital and community isolates of identical species, he concluded that hospital isolates were only slightly more resistant. However, Kayser (7) and Shah et al. (12) have concluded differently, saying that hospital isolates of Escherichia coli, Klebsiella spp. and Staphylococcus aureus were more resistant than community strains. In addition, many of the species causing classical hospital-acquired infection in immunosuppressed patients and which rarely cause community-acquired infections, e.g. Pseudomonas spp., frequently are multi-resistant.

\section{Hospital Infection}

There is increasing interest in hospital infection rates as indicators of the quality of health care, and surveillance is now the norm (13). Commonly accepted incidence figures are around 5-10\%, but they vary widely, not the least because of problems of definition of resistance and differentiating between colonisation and infection. However, as colonisation is very often a prerequisite for infection, this latter difficulty may not be such a problem when studying multiply resistant organisms. Urinary tract infection, usually related to an indwelling catheter, is the most common nosocomial infection and cause of gram-negative bacteraemia (14) and is due increasingly to multiply resistant organisms. Nosocomial pneumonia, post-operative wound infection and vascular access infection are next most common, all with variable but similar incidences. Nosocomial pneumonia is frequently due to MRGNB, but these organisms are rarely involved in vascular access infection, and while frequently colonising post-operative wounds and burns, they are involved less frequently in invasive infection than Staphylococcus aureus, haemolytic streptococci and anaerobes. They are probably also less common in neutropenic patients than previously thought (15). 
Table 1: Number of ICU patients with one or more infection (unpublished data of the European study for Prevalence of Infection in Intensive Care. [EPIIC]).

\begin{tabular}{lc}
\hline Type of infection & No. (\%) of patients \\
\hline All infections & $4,501(44.8)$ \\
ICU acquired & $2,064(20.6)$ \\
Hospital acquired & $975(9.7)$ \\
Community acquired & $1,376(13.7)$ \\
\hline
\end{tabular}

Table 2: Frequency of infection types in patients with one or more ICU-acquired infection (European study for Prevalence of Infection in Intensive Care [EPIIC]).

\begin{tabular}{ll}
\hline Type of Infection & No. (\%) of patients \\
\hline Pneumonia & $967(46.9)$ \\
Lower respiratory tract infection & $638(17.8)$ \\
Sepsis or bloodstream infection & $368(17.8)$ \\
UTI or related infection & $363(17.6)$ \\
Wound infection & $142(6.9)$ \\
\hline
\end{tabular}

Infection rates in ICUs are much higher and the spectrum of infection different from that in other hospital wards. (Tables 1 and 2). This data, as yet unpublished, is taken from a preliminary analysis of figures from a European survey of ICUs carried out by the European Prevalence of Infection in Intensive Care (EPIIC) study. Ventilator-associated respiratory infection is the most common, although the incidence of ventilator-associated pneumonia (VAP) versus purulent tracheobronchitis is an important unresolved issue due to the difficulties in differentiating pneumonia from other causes of radiological opacities. The prognosis of tracheobronchitis and the frequency of progression to VAP are unclear but important issues, both for outcome and treatment and also for studies on incidence and prevention of infection (16).

\section{Cross-Infection Problems and Outbreaks with Multiply Resistant Gram-Negative Bacteria}

Clearly, one of the major risk factors for a patient to become colonised or infected with MRGNB is to be admitted to a unit where such a strain exists and to be the victim of a breakdown in infection control procedures. Perhaps such lapses in technique are inevitable, as such organisms are ubiquitous in the environment of hospitals, espe- cially in ICUs, and once present they are very difficult to eradicate. Rigorous control of technique is needed at all times to prevent transfer from patient to patient. Frequently, however, units are very busy and short staffed and often the understanding of infection control techniques is confused or not considered important. Compliance with hand washing, for example, probably is rarely more than $50 \%$ (17). The consequences and costs of such cross infection need to be better studied and explained, but surely new solutions are needed when still we have to extol the virtues of handwashing almost 200 years after Semelweiss.

The recent literature is full of reports of lapses in infection control technique leading to outbreaks of MRGNB that are too numerous to mention here. Clearly, many of the risk factors are interrelated, some only being associated or compounding factors, but multivariate analysis has been employed only rarely in assessing the risk factors (18, $19)$. Some key factors are admission when colonised with a resistant organism (20), antibiotic exposure with loss of colonisation resistance (21), duration of stay (22), cross infection (23), ventilation (24) and catheterisation $(18,25)$. Immunosuppression and invasive diagnostic and therapeutic procedures lead inevitably to increased colonisation with resistant organisms, and from there infection is established easily by what are often less virulent organisms. Use of disinfectants, antiseptics and other topical agents has long been known to be a hazard if these solutions are not prepared fresh each day. Many MRGNB colonise such solutions readily, and there may be links between resistance to antibiotics and these agents (26). Colonisation of these and other damp environmental sites in the hospital provides a ready source for contamination of patients with the slightest breakdown in infection control procedures $(27,28)$. Transfer is accomplished through the hands of staff and inadequately disinfected or sterilised multi-use containers, but transmission through intermediate vehicles such as curtains, staff clothes and sheets also probably occurs.

Weinstein (29) has reviewed classical infection control techniques and the reasons they fail. One reason is thought to be that many bacteria causing nosocomial infection are endogenous in origin. This has led to the evaluation of selective digestive decontamination (SDD) as an infection control procedure. This has been advocated both as a means of reducing the oropharyngeal and enteric load of the host's commensal gram-negative bacilli, which may be responsible for up to $50 \%$ of 
ICU infections, and also as a means of controlling outbreaks of MRGNB. It does, however, remain a research procedure only, with no proven benefits and the potential risks of increased antibiotic exposure and development of resistance $(30,31)$.

\section{Resistance Patterns of Multiply Resistant Gram- Negative Bacilli}

Many gram-negative bacilli, particularly Pseudomonas spp., are innately resistant to many antibiotics, and there is no doubt that we have seen an increase in infection by these bacteria. A greater problem is resistance acquisition by more traditional hospital pathogens such as Klebsiella spp. Plasmid-mediated enzymes, which inactivate $\beta$ lactams, are now ubiquitous and, more worryingly, modification of some of these enzymes (TEM and SHV types) produces resistance to third-generation cephalosporins. These enzymes are becoming common, with up to $40 \%$ of Klebsiella spp. in some units now being resistant to ceftazidime (3). Similarly, monobactam, cephamycin and third-generation cephalosporin resistance is now commonplace in inducible Enterobacteriaceae and, if not present at the start of therapy, is likely to emerge by selection of induced strains during therapy (32). This has led some authorities to call for alternative regimens to be used in these situations. If these strains also lose the $D_{2}$ porin giving carbapenem permeability, they can become carbapenem resistant, and such strains have been described occasionally (33). In addition, carbapenemases are being described increasingly, although at the present they are still rare (33).

The development of multiple resistance to more than one class of agent is more problematic. Table 3 lists some recent reports describing development of resistance to more than one class of agents. Clearly, the 4-fluoroquinolones are heavily incriminated, probably through alteration of bacterial outer membrane proteins. It is also clear, however, that different resistance determinants can accumulate on the same plasmid. Plasmids can code not only for broad-spectrum penicillin and cephalosporin resistance but also for aminoglycoside and cotrimoxazole resistance (5). The mechanism for this seems to be an association between antibiotic-resistance-determining sequences and integration sequences which encourage not only the addition of further resistance determinates but their spread to other plasmids as well.

\section{Antibiotics as a Risk Factor for Selection of Mul- tiply Resistant Gram-Negative Bacteria}

Inevitably, the increasing use of extended-spectrum agents selects for increasingly resistant organisms, and this is never more evident than in hospitals, particularly in ICUs. Many studies now show that antimicrobial sensitivity patterns are a negative imprint of the agents used, resistance relating to per capita consumption in the commun-

Table 3: Risk factors for colonisation/infection with multiply resistant gram-negative bacteria.

\begin{tabular}{|c|c|c|c|}
\hline Patient group & Risk factors & Organism & Reference \\
\hline Community & $\begin{array}{l}\text { 4-fluoroquinolone, } \\
\text { tetracycline, } \\
\text { chloramphenicol use }\end{array}$ & $\begin{array}{l}\text { 4-fluoroquinolone resistant } \\
\text { Escherichia coli }\end{array}$ & 34 \\
\hline Not stated & 4-fluoroquinolone use & $\begin{array}{l}\text { 4-fluoroquinolone, } \\
\text { carbapenem resistant } \\
\text { Pseudomonas aeruginosa }\end{array}$ & 35 \\
\hline General hospital & ciprofloxacin use & $\begin{array}{l}\text { third generation, } \\
\text { cephalosporin, aminoglycoside, } \\
\text { azlocillin, cotrimoxazole, } \\
\text { minocycline resistant Acinetobacter calcoaceticus }\end{array}$ & 36 \\
\hline Community & $\begin{array}{l}\text { chloramphenicol, } \\
\text { trimethoprim, } \\
\text { nalidixic acid use }\end{array}$ & $\begin{array}{l}\text { Multiply-resistant Klebsiella pneumoniae, } \\
\text { Acinetobacter calcoaceticus, } \\
\text { inducible enterobacteriaceae }\end{array}$ & 37 \\
\hline ICU & excessive use of antibiotics & Pseudomonas aeruginosa & 38 \\
\hline
\end{tabular}


ity and in the hospital (6), resistance patterns often altering upon removal of selecting agents from use $(39,40)$. The situation is complicated, however, by the environment, the potential for cross infection and the ability of one agent to select for resistance to another unrelated agent through a common resistance mechanism or a plasmid sharing separate resistance determinants. Widespread use of third-generation cephalosporins in hospitals and homes for the elderly, in association with cross infection, is leading to outbreaks of expanded-spectrum $\beta$-lactamases (3). In addition, there appears to be a strong correlation between the emergence of inducible Enterobacteriaceae in intensive care and the use of third-generation cephalosporins $(32,41)$, and these isolates are more likely to be resistant to these agents (42). In a recent UK survey these isolates accounted for approximately one-third of all blood isolates from ICU patients (personal communication, N. Deaney, Merck Sharp \& Dohme).

There is little evidence to incriminate any one particular group of agents as being more likely to lead to the emergence of resistant organisms during treatment (43). The combination of an aminoglycoside with a cephalosporin did not appear to affect the emergence of resistant strains in one recent study (42), although there are experimental reasons and some clinical evidence as to why combination therapy should give some benefit (43). Similarly, inadequate dosing (likely to lead to suboptimal antibiotic levels) and high-inoculum infections encourage the emergence of more resistant strains. In addition, where the infecting organisms can be protected from antibiotic, such as in an abscess or in infections associated with biofilm on a prosthesis or in the respiratory tract of patients with cystic fibrosis, low concentrations of antibiotic may be present despite apparently adequate dosing, leading to selection of resistant variants. At present, imipenem seems to have an advantage in that resistance arising during treatment is not a problem with the inducible Entero- bacteriaceae (Table 4). The ecological problem of development of resistance in the large reservoirs of commensal bacteria in the gut and on the skin seems to have been addressed only recently. Many studies now show a strong link between the use of agents and subsequent development of clinical problems with gram-negative bacilli resistant to these agents, whether they be aminoglycosides (44-46), $\beta$-lactams $(44,47,48)$ or 4 -fluoroquinolones (49-52).

\section{Resistance Rates}

Antibiotic resistance is clearly a worldwide problem, although its incidence appears to vary markedly from country to country and institution to institution. Data from different studies is often difficult to compare due to different definitions of resistance (53). Coordinated worldwide studies such as that from the World Health Organisation (54) and the Centers for Disease Control, Atlanta (55), are therefore essential. Early warning of some of our current resistance problems might have allowed containment of epidemic plasmids and delayed or stopped the spread of resistant organisms (56).

Data on the incidence of MRGNB is actually very scarce in the literature, most studies concentrating on single resistance mechanisms for each organisms. One recent study of ICU isolates suggested that in the UK about $30 \%$ of gram-negative clinical isolates were resistant to at least two of the four major antibiotic groups (aminoglycosides, 4-fluoroquinolones, third-generation cephalosporins and carbapenems) and $10 \%$ to three or all of the groups (Table 5).

The hope that bacteria carrying additional genetic information would be at a disadvantage is clearly not applicable as long as the information carries survival value. Removal of the selection pressure

Table 4: Development of resistance in relation to bacterial species during treatment (from Reference 30).

\begin{tabular}{lrccccc}
\hline Organism & \multicolumn{5}{c}{ Percentage of strains $(\mathrm{n})$ that developed resistance } \\
\cline { 2 - 7 } & Penicillins & Cephalosporins & Latamoxef & Imipenem & Ciprofloxacin & Aminoglycoside \\
\hline $\begin{array}{l}\text { P.aeruginosa } \\
\text { Enterobacter, }\end{array}$ & $22.0(50)$ & $18.3(115)$ & $23.9(71)$ & $24.5(59)$ & $16.7(221)$ & $21.1(57)$ \\
Citrobacter, & $7.7(26)$ & $9.9(81)$ & $12.1(58)$ & $0(38)$ & $5.9(34)$ & $13.6(29)$ \\
Serratiaspp. & & & & & & \\
\hline
\end{tabular}


Table 5: Multiple resistance to antibiotics in gram-negative bacilli isolated from ICUs (personal communication, N. Deaney, Merck Sharp \& Dohme).

\begin{tabular}{lcc}
\hline Resistance pattern & No. of strains & Percent resistant \\
\hline CAZ & 137 & 30.0 \\
CAZ + GM & 73 & 16.0 \\
CAZ + CIP & 43 & 9.4 \\
CAZ + IMP & 43 & 9.4 \\
CAZ + GM + CIP & 11 & 2.4 \\
CAZ + GM + IMP & 13 & 2.9 \\
CAZ + GM + IMP + CIP & 16 & 3.5 \\
\hline
\end{tabular}

CAZ: ceftazidime; GM: gentamicin; CIP: ciprofloxacin; IMP: imipenem.

by reducing the use of an agent often leads to increased sensitivity to that agent $(12,53)$, but where multiple resistance mechanisms are linked, continued use of one agent may lead to persistence of all resistant mechanisms (11). Thus, at least in the hospital setting, once there is a resistance problem, it may be difficult to reverse the trend, even with major restrictions on antibiotic use (57). Complete cessation of antibiotic use (rarely possible) can be successful (39), but the situation may be further complicated by the selection power of antiseptics, disinfectants and cross infection.

\section{Prevention of Selection of Multiply Resistant Gram-Negative Bacteria}

Per capita antibiotic consumption seems to be increasing relentlessly (41) (author's unpublished observation). This does not appear to be linked to increased patient numbers in our institution, although increased immunosuppression and opportunistic infection may be part of the explanation. In general, however, while new agents may replace some older agents, the use of many older agents is also increasing or at least remaining stable, suggesting that many more patients are receiving antibiotics (41). There is ample evidence that much of this use is inappropriate $(58,59)$. For example, duration of perioperative prophylaxis is frequently prolonged unnecessarily. Some degree of restriction of antibiotic use clearly is required in hospitals, other institutions and in the community. Until now, antibiotic use has been relatively unrestricted, with many agents being available for purchase over the counter in some countries, while in others, physicians actually receive financialincentives to prescribe new (and usually more expensive) broad-spectrum agents. There has even been a recent call in the UK to allow patients to purchase certain antibiotics without prescription to give patients greater control over their own health. Clearly, however, antibiotics are not like other drugs in that they have a societal perspective: the development and spread of resistance.

Surveys from the UK (unpublished data) and the Netherlands (58) suggest that the majority of hospitals in these countries do have formularies and policies in place to control antibiotic use, although evidence of their efficacy is more difficult to establish. There is, however, widespread confidence in their use, but this is not matched by the number of non-formulary agents that are prescribed. The British government recently has introduced local and national initiatives for the education of prescribers, which may improve matters. The Infectious Diseases Society of America (60) and the British Society for Antimicrobial Chemotherapy (unpublished data) both have guidelines for antibiotic use and control in hospitals, and guidelines for treatment and prophylaxis of specific diseases are proving popular (61). National formularies can also be of benefit for developing countries (62).

Diagnostic techniques also must be improved to enable specific therapy to be given. One particular area of concern is in ICUs. The majority of infections are ventilatory associated, but the frequent inability to differentiate bacterial pneumonia from other causes of radiological opacities (63) leads to excessive use of antibiotics, usually broad-spectrum agents. One attempt to address this problem has been the use of SDD. Although not used commonly (EPIIC study, unpublished), SDD is advocated enthusiastically by its proponents, both for reducing VAP and other infections with gram-negative bacteria in selected patients and for controlling outbreaks of MRGNB. Unfortunately, as explained above, it is difficult to interpret the results of the many studies to date for various reasons, including the major problem of diagnosing VAP (63). In addition, the historical experience of using such regimens is fraught with development of infection with organisms resistant to the chosen regimens. While this does not seem to have been a major problem with SDD to date, surely it must be inevitable (30).

Even if SDD is proved ultimately to be efficacious in certain groups of patients, the benefits, whether they be prevention of VAP and reduced ICU stay or even reduced mortality, must be balanced 
against the likely ecological damage of such widespread, relatively undirected, prophylaxis. Insufficient assessment of such aspects in the past has led to widespread use of antibiotic prophylaxis in leukaemic patients with no proven benefits but with the consequences of increased resistance and infection/colonisation with resistant strains (64).

\section{Conclusions}

Control of cross infection by traditional measures surely must be the mainstay of our attempts in the near future to stop the spread of MRGNB selected by antimicrobial use. Unfortunately, the situation is increasingly complex in hospitals, particularly in ICUs, but the basic principles remain and must be clearly taught, understood and acted upon. It is important to resist expensive preventative measures of dubious value. The cost of such measures should be evaluated and compared with the costs of infection with MRGNB. Early warning of problems of resistance on a worldwide basis must be achieved by improved surveillance. Many of the resistance problems recently encountered seem to have arisen with little warning. Antibiotic use must be restricted further, both in hospitals and in the community, and new educational initiatives introduced to encourage better prescribing. In 1986 Kresken and Wiedemann (1) wrote, "The finding of low levels of resistance to new antibiotics on the one side and higher resistance levels for older drugs on the other side should not lead to the conclusion that the former may be used more widely. The new drugs should be reserved for essential indications and the older drugs for approved uses. By these means resistance problems should continue to be controlled." This is equally applicable in 1994.

\section{References}

1. Kresken M, Wiedemann B: Development of resistance in the past decade in central Europe. Joumal of Antimicrobial Chemotherapy 1986, 18, Supplement C: 235-242.

2. Sanders CC, Sanders WE Jr: $\beta$-lactam resistance in gram negative bacteria: global trends and clinical impact. Clinical Infectious Diseases 1992, 15: 824-839.

3. Payne DJ, Amyes SGB: Transferable resistance to extended spectrum $\beta$ lactams: a major threat or a minor inconvenience? Journal of Antimicrobial Chemotherapy 1991, 27: 255-261.
4. Fung-Tome J, Kolek B, Bonner DP: Ciprofloxacin-induced, low level resistance to structurally unrelated antibiotics in Pseudomonas aeruginosa and methicillin resistant Staphylococcus aureus. Antimicrobial Agents and Chemotherapy 1993, 37: 1289-1296.

5. Jacoby GA, Archer GL: New mechanisms of bacterial resistance to antimicrobial agents. New England Journal of Medicine 1991, 324: 601-612.

6. Cohen ML: Epidemiology of drug resistance: implications for a post antimicrobial era. Science 1992, 257: 1050-1055.

7. Kayser FH: Evolution of resistance in microorganisms of human origin. Veterinary Microbiology 1993, 35: 257-267.

8. Mandal BK: Epidemic cholera due to a novel strain of $V$. cholerae non-O1 - the beginning of a new pandemic? Journal of Infection 1993, 27: 115-117.

9. Obaseiki-Ebor EE, Akerele JO, Ebea PO: A survey of antibiotic outpatient prescribing and antibiotic self medication. Joumal of Antimicrobial Chemotherapy 1987, 20: 759-763.

10. Thamlikitkul V: Antibiotic dispensing by drug store personnel in Bangkok, Thailand. Journal of Antimicrobial Chemotherapy 1988, 21: 125-131.

11. McGowan JE, Hall EC, Parrott PL: Antimicrobial susceptibility in gram-negative bacteremia: are nosocomial isolates really more resistant? Antimicrobial Agents and Chemotherapy 1989, 33: 1855-1859.

12. Shah PM, Schäfer V, Knothe H: Medical and veterinary use of antimicrobial agents: implications for public health. A clinician's view on antimicrobial resistance. Veterinary Microbiology 1993, 35: 269-274.

13. Chaudhuri AR: Infection control in hospitals: has its quality-enhancing and cost-effective role been appreciated? Journal of Hospital Infection 1993, 25: 1-6.

14. Bergogne-Bérézin E, Decré D, Joly-Guillou ML: Opportunistic nosocomial multiply resistant bacterial infections - their treatment and prevention. Joumal of Antimicrobial Chemotherapy 1993, 32, Supplement A: 39-47.

15. Davey P: Antibacterial regimens for neutropenic patients in intensive care. Intensive Care Medicine 1990, 16: 80-87.

16. Craven DE: Use of selective decontamination of the digestive tract. Annals of Internal Medicine 1992, 117: 609611.

17. Edmond MB, Wenzel RP: Ethical considerations in the use of subliminal stimulation to improve handwashing compliance: scientific utility versus autonomy of the individual. Infection Control and Hospital Epidemiology 1993, 14: 107-109.

18. Culver DH, Horan TC, Gaynes RP, Marton WJ, Jarvis WR, Emori TG, Banerjee SN, Edwards JR, Tolson JS, Henderson TS, Hughes JM: Surgical wound infection rates by wound class, operative procedure and patient risk index. American Journal of Medicine 1991, 91, Supplement 3B: 152-157.

19. Hanson LC, Weber DJ, Rutala WA: Risk factors for nosocomial pneumonia in the elderly. American Journal of Medicine 1992, 92: 161-166.

20. Olson B, Weinstein RA, Nathan C, Chamberlin W, Kabins SA: Epidemiology of endemic Pseudomonas aeruginosa: why infection control efforts have failed. Journal of Infectious Diseases 1984, 150: 808-816.

21. Olson B, Weinstein RA, Nathan $\mathbf{C}$, Chamberlin W, Kabins SA: Occult aminoglycoside resistance in Pseudomonas aeruginosa: epidemiology and implications for therapy and control. Journal of Infectious Diseases 1985, 152: 769-774. 
22. Kerver AJH, Rommes JH, Mevissen-Verhage EAE, Hulstaert PF, Vos A, Verhoef J, Wittebol P: Colonization and infection in surgical intensive care patients - a prospective study. Intensive Care Medicine 1987, 13: 347-351.

23. Widmer AF, Wenzel RP, Trilla A, Bale MJ, Jones RN, Docbbeling BN: Outbreak of Pseudomonas aeruginosa infections in surgical intensive care unit: probable transmission via hands of a health care worker. Clinical Infectious Diseases 1993, 16: 372-376.

24. Pokrywka M, Viazanko $\mathbf{K}$, Medvick J, Knabe $\mathbf{S}$, McCool S, Pasculle AW, Dowling JN: A Flavobacterium meningosepticum outbreak among intensive care patients. American Journal of Infection Control 1993, 21: 139-145.

25. Kiehn TE, Armstrong D: Changes in the spectrum of organisms causing bacteremia and fungemia in immunocompromised patients due to venous access devices. European Journal of Clinical Microbiology and Infectious Diseases 1990, 9: 869-872.

26. Jacoby GA, Sutton L: Properties of plasmids responsible for production of extended spectrum $\beta$-lactamases. Antimicrobial Agents and Chemotherapy 1991, 35: 164-169.

27. Gorman L, Sanai L, Notman AW, Grant IS, Masterton RG: Cross infection in an intensive care unit by Kleb. siella pneumoniae from ventilator condensate. Journal of Hospital Infection 1993, 23: 27-34.

28. Pegues DA, Shireley LA, Riddle CF, Anderson RL, Vess RW, Hill BC, Jarvis WR: Serratia marcescens surgical wound infection following breast reconstruction. American Journal of Medicine 1991, 91, Supplement 3B: 173-178.

29. Weinstein RA: Epidemiology and control of nosocomial infections in adult intensive care units. American Journal of Medicine 1991, 91, Supplement 3B, 179-184.

30. Gould IM: Selective parenteral and enteral antisepsis regimen (SPEAR). British Journal of Hospital Medicine 1990, 44: 209-287.

31. Atkinson SW: Selective decontamination of the gut. British Medical Journal 1993, 306: 286-287.

32. Fynn DM, Weinstein RA, Nathan C, Gaston MA, Kabins SA: Patients' endogenous flora as the source of "nosocomial" enterobacter in cardiac surgery. Journal of Infectious Diseases 1987, 156: 363-368.

33. Livermore DM: Carbapenemases: The next generation of $\beta$-lactamases? ASM News 1993, 59: 129-135.

34. Trallero EP, Urbieta M, Jimenez D, Garcia-Arenzana Cilla G: Ten year survey of quinolone resistance in Escherichia coli causing urinary tract infections. European Journal of Clinical Microbiology and Infectious Diseases 1993, 12: 349-351.

35. Aubert G, Pozzetto B, Dorche G: Emergence of quinolone-imipenem cross-resistance in Pseudomonas aeruginosa after fluoroquinolone therapy. Journal of Antimicrobial Chemotherapy 1992, 29: 307-312.

36. Shalit I, Dan M, Gutman R, Gorea A, Berger SA: Cross resistance to ciprofloxacin and other antimicrobial agents among clinical isolates of Acinetobacter calcoaceticus biovar anitratus. Antimicrobial Agents and Chemotherapy 1990, 34: 494-495.

37. Gutmmnn L, Williamson R, Moreau N, Kitzis M, Collatz E, Acar JF, Goldstein FW: Cross-resistance to nalidixic acid, trimethoprim and chloramphenicol associated with alterations in outer membrane proteins of Klebsiella, Enterobacter and Serratia. Journal of Infectious Diseases 1985, 151: 501-507.
38. Allen KD, Bartzokas CA, Graham R, Gibson MF, Gilbertson AA: Acquisition of endemic Pseudomonas aeruginosa on an intensive therapy unit. Journal of Hospital Infection 1987, 10: 156-164.

39. Price JE, Sleigh JD: Control of infection due to Klebsiella aerogenes in a neuro-surgical unit by withdrawal of all antibiotics, Lancet 1970, ii: 1213-1215.

40. Gerding DN, Larson TA, Hughes RA, Weiler M, Shanholtzer C, Peterson LR: Aminoglycoside resistance and aminoglycoside usage: ten years of experience in one hospital. Antimicrobial Agents and Chemotherapy 1991, 35: $1284-1290$.

41. Pallares R, Dick R, Wenzel RP, Adams JR, Nettleman MD: Trends in antimicrobial utilization at a tertiary teaching hospital during a 15 year period (1978-1992). Infection Control and Hospital Epidemiology 1993,14: 376-382.

42. Chow JW, Fine MJ, Shlaes DM, Quinn JP, Hooper DC, Johnson MP, Ramphal R, Wagener M, Miyashim DK, Yu VL: Enterobacter bacteremia: clinical features and emergence of antibiotic resistance during therapy, Annals of Internal Medicine 1991, 115: 585-590.

43. Milatovic D, Braveny I: Development of resistance during antibiotic therapy. European Journal of Clinical Microbiology 1987, 6: 234-244.

44. Courcol RJ, Pinkas M, Martin GR: A seven year survey of antibiotic susceptibility and its relationship with usage. Journal of Antimicrobial Chemotherapy 1989, 23: 441-451.

45. Friedland IR, Funk E, Khoosal M, Klugman KP: Increased resistance to amikacin in a neonatal unit following intensive amikacin usage. Antimicrobial Agents and Chemotherapy 1992, 6: 1596-1600.

46. Gerding DN, Larson TA, Hughes RA, Weiler $M$, Shanholtzer C, Peterson LR: Aminoglycoside resistance and aminoglycoside usage: ten years of experience in one hospital. Antimicrobial Agents and Chemotherapy 1991, 35; $1284-1290$.

47. Vurma-Rapp U, Kayser FH, Hadorn K, Wiederkehr F: Mechanism of imipenem resistance acquired by three Pseudomonas aeruginosa strains during imipenem therapy. European Journal of Clinical Microbiology and Infectious Diseases 1990, 9: 580-587.

48. Mulgrave L: The changing ecology of hospital bacteria and the selective role of cephalosporins. Epidemiology and Infection 1991, 106: 121-132.

49. Aubert G, Levy PP, Ros A, Meley R, Meley B, Bourge $A$, Dorche $G$ : Changes in the sensitivity of urinary pathogens to quinolones between 1987 and 1990 in France. European Joumal of Clinical Microbiology and Infectious Diseases 1992, 11: 475-477.

50. Jonsson M, Walder M, Forsgren A: First clinical isolate of highly fluoroquinolone-resistant Escherichia coli in Scandinavia. European Journal of Clinical Microbiology and Infectious Diseases 1990, 9: 851-853.

51. Aguiar JM, Chacon J, Canton R, Baquero F: The emergence of highly fluoroquinolone-resistant Escherichia coli in community-acquired urinary tract infection. Journal of Antimicrobial Chemotherapy 1992, 29: 349-350.

52. Somolinos N, Arranz R, Del Rey MC, Jiménez ML. Superinfections by Escherichia coli resistant to fluoroquinolones in immunocompromised patients. Journal of Antimicrobial Chemotherapy 1992, 30: 730-731.

53. Wiedemann B: Monitoring of resistant organisms in man and identification of their origin. Veterinary Microbiology 1993, 35: 275-284.

54. O'Brien TF: Global surveillance of antibiotic resistance. New England Joumal of Medicine 1992, 326: 339-340. 
55. Newsnote: Worldwide surveillance of infectious disease agents and drug resistance. Alliance for the Prudent Use of Antibiotics Newsletter 1993, 11: 5-7.

56. O'Brien TF, Mayer KH, Gilleece E, Syvanen M, Hopkins JD: Intercontinental spread of a new antibiotic resistance gene on an epidemic plasmid. Science 1985, 230: $87-88$.

57. Legakis NJ, Tzouvelekis LS, Tsakris A, Legakis JN, Vatopoulos $\mathrm{C}$ : On the incidence of antibiotic resistance among aerobic gram-negative rods isolated in Greek hospitals. Journal of Hospital Infection 1993, 24: 233-237.

58. Stobberingh E, Janknegt $\mathbf{R}$, Wijnands G: Antibiotic guidelines and antibiotic utilization in Dutch hospitals. Journal of Antimicrobial Chemotherapy 1993, 32: 153-161.

59. Halls GA: The management of infections and antibiotic therapy: a European survey. Journal of Antimicrobial Chemotherapy 1993, 31: 985-1000.
60. Infectious Diseases Society of America: Report from the Antimicrobial Agents Committee. Journal of Infectious Diseases 1987, 156: 700-705.

61. Gould IM: Current prophylaxis for prevention of infective endocarditis. British Dental Journal 1990, 169, 409-410.

62. Hogerzeil HV, Walker GJA, Sallami AO, Fernando G: Impact of an essential drugs programme on availability and rational use of drugs. Lancet, 1989, i: 141-142.

63. Guerra LF, Baughman RP: Use of bronchoalveolar lavage to diagnose bacterial pneumonia in mechanically ventilated patients. Critical Care Medicine 1990, 18: 169-173.

64. Bow EJ, Ronald AR: Antibacterial chemoprophylaxis in neutropenic patients - where do we go from here? Clinical Infectious Diseases 1993, 17: 333-337. 